\title{
The Polarized-Target
}

System for the SpinQuest Experiment at Fermilab

Zulkaida Akbar

(For SpinQuest Collaboration)

University of Virginia

2020 Fall Meeting of the APS Division of Nuclear Physics

$10 / 31 / 2020$

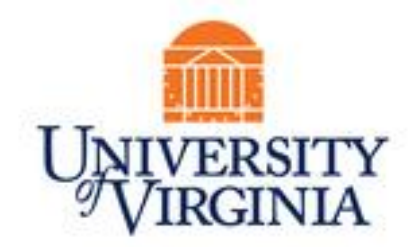

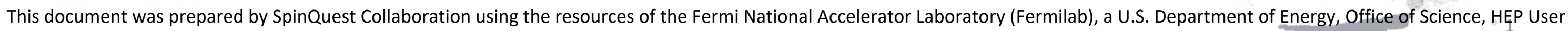
Facility. Fermilab is managed by Fermi Research Alliance, LLC (FRA), acting under Contract No. DE-AC02-07CH11359. 


\section{Outline}

- Introduction

- Microwave System

- Target Materials

- Superconducting-Magnet System

- Cryogenics

- Nuclear-Magnetic Resonances (NMR) system

- Summary 


\section{Introduction: SpinQuest Experiment at Fermilab}

Physics goal:

$\square$ Perform the first measurement of the Sivers asymmetry in Drell-Yan pp scattering from the sea quarks

A non-zero Sivers asymmetry from SpinQuest is "smoking gun" evidence for sea quark Orbital-Angular Momentum
Require a transversely polarized target capable of both high polarization and integrated luminosity:

Attempt to push the proton beam intensity frontier on a solid polarized target

$\square$ Use the longest target cell (and most volume) ever ran in a $1 \mathrm{~K}$ evaporation polarized target system

$\square$ Highest cooling power DNP (Dynamic Nuclear Polarization) target in the world due to the high pumping rate and the refrigerator. 


\section{Introduction:}

\section{SpinQuest Vs BNL Experiment on polarized PP scattering}

Time-Structure of the Beam

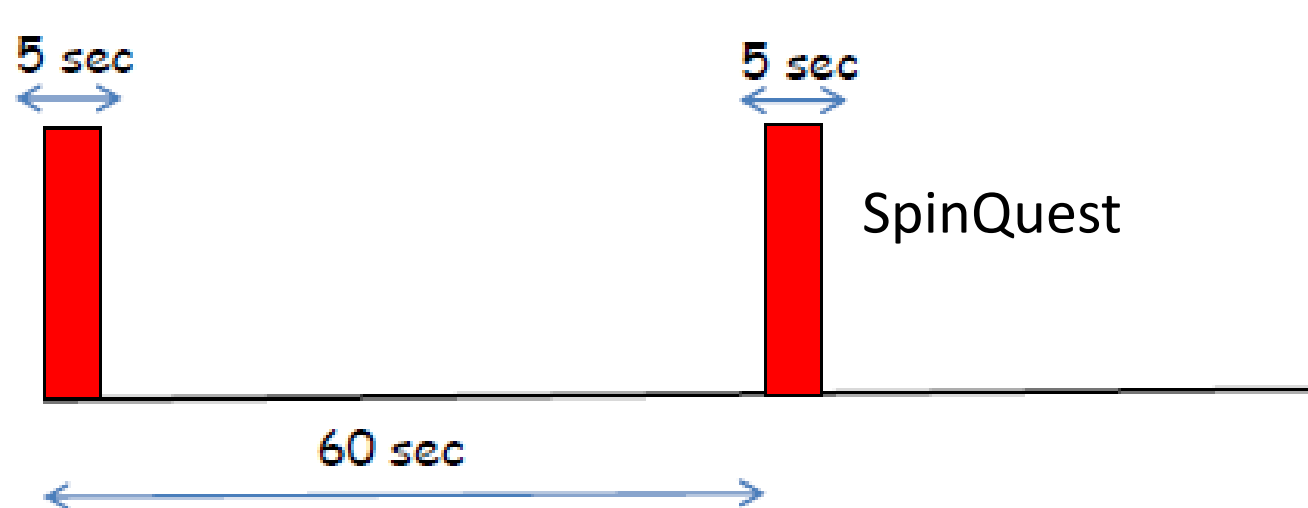

$1 \mathrm{sec}$

Time-Structure of the Beam

\begin{tabular}{|l|l|l|} 
& SpinQuest & BNL \\
\hline Beam Energy & $120 \mathrm{GeV}$ & $24 \mathrm{GeV}$ \\
\hline Intensity & $\sim 5 \times 10^{12} /$ spill & $2 \times 10^{11} /$ spill \\
\hline Spill length & 4.4 second & 1 second \\
\hline Target length & $8 \mathrm{~cm}$ & $3 \mathrm{~cm}$
\end{tabular}

$\mathrm{BNL}$ 


\section{Introduction: \\ How do we obtain significant nucleon polarization?}

Brute-Force Method:

- Use high-B at low-T via zeeman-splitting mechanism

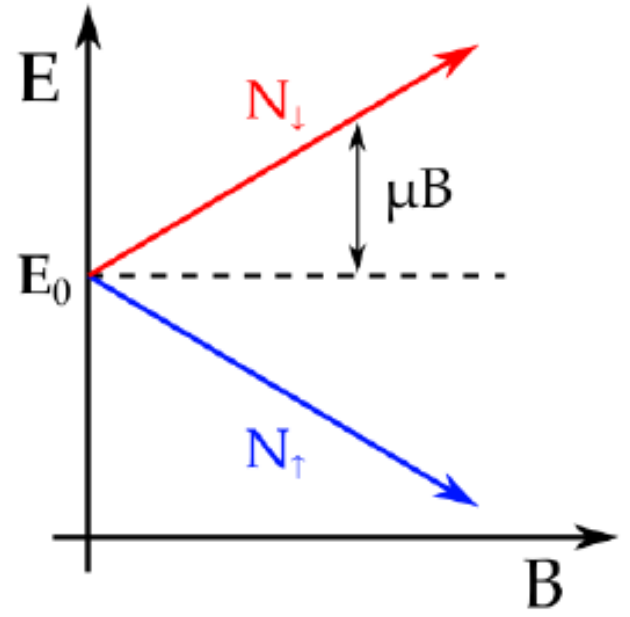

Courtesy of James Maxwell
- Degree of polarization at thermal equilibrium

$$
P=\tanh \left(\frac{\mu B}{k T}\right)
$$

- Proton has small magnetic moment

$$
\mu_{e} \approx 660 \mu_{p}
$$

- At $B=5$ Tesla $\& T=1 \mathrm{~K}$

$$
P_{e}=\sim 98 \%, P_{p}=0.51 \%
$$

- We need a better method!

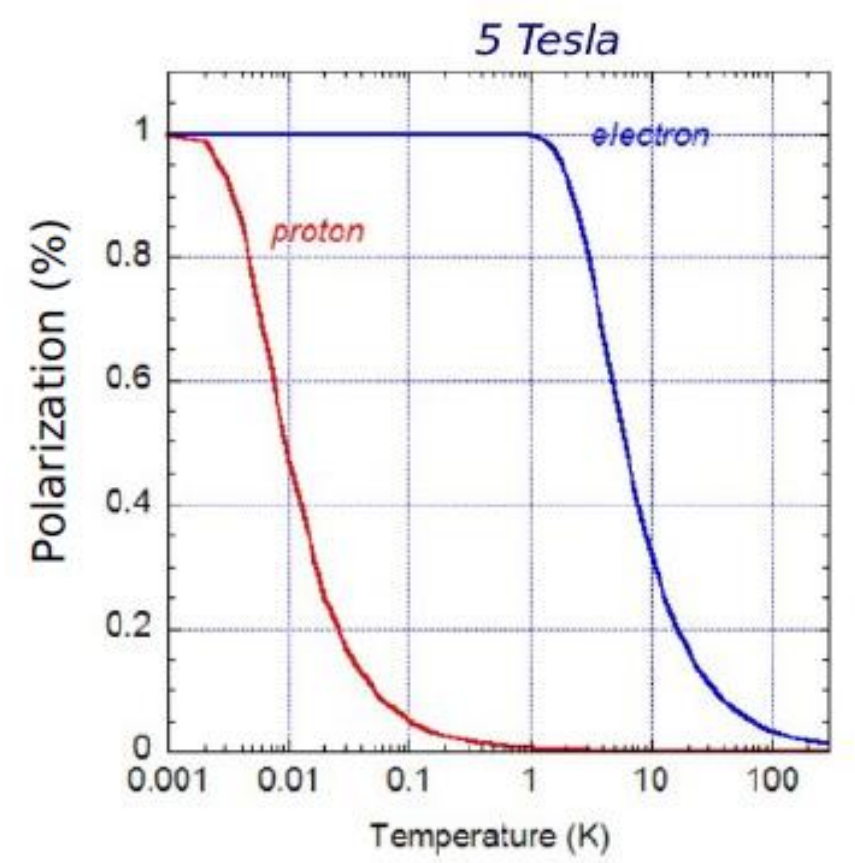




\section{Introduction: \\ How do we obtain significant nucleon polarization?}

Dynamic-Nuclear Polarization (DNP):

- The coupling between (unpaired) electron \& proton introduces hyperfine splitting

$H=-\mu_{e} B-\mu_{p} B+H_{S S}$

- Applying an RF-field at the correct frequency, we can drive the nucleons state into desired proton-state
- The disparity in relaxation times between the electron (ms) and proton (tens of minutes) at $1 \mathrm{~K}$ is crucial to continue proton polarization

- Allow to achieve proton polarization of $>90 \%$

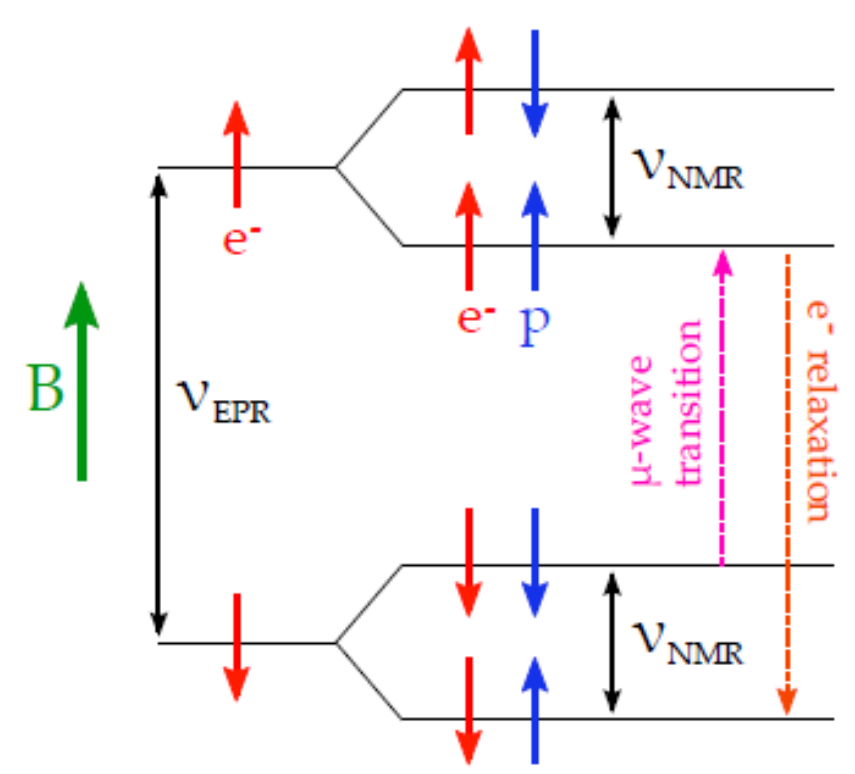




\section{Dynamic Nuclear Polarization}

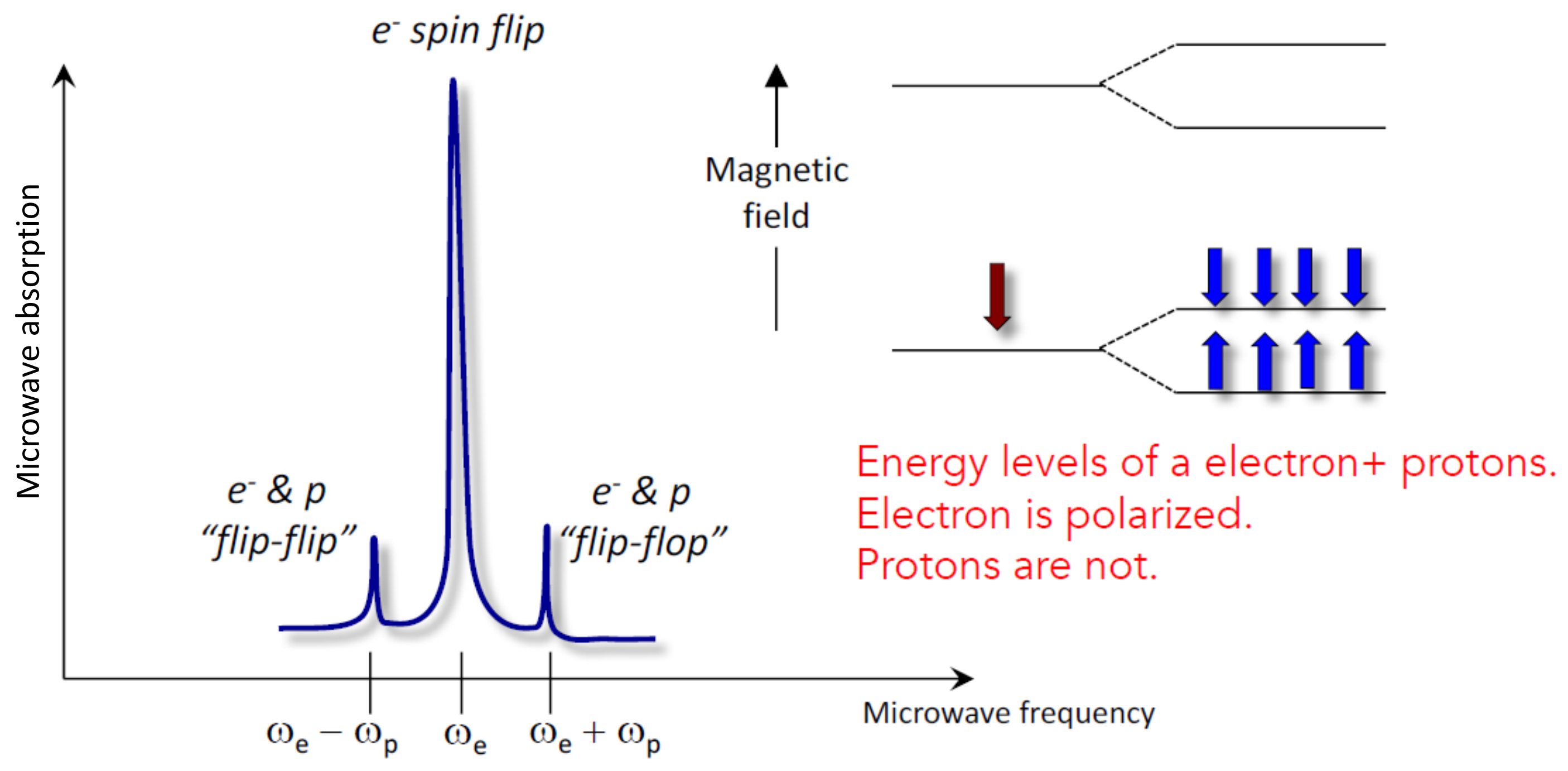

Courtesy of Chris Keith 


\section{Dynamic Nuclear Polarization}

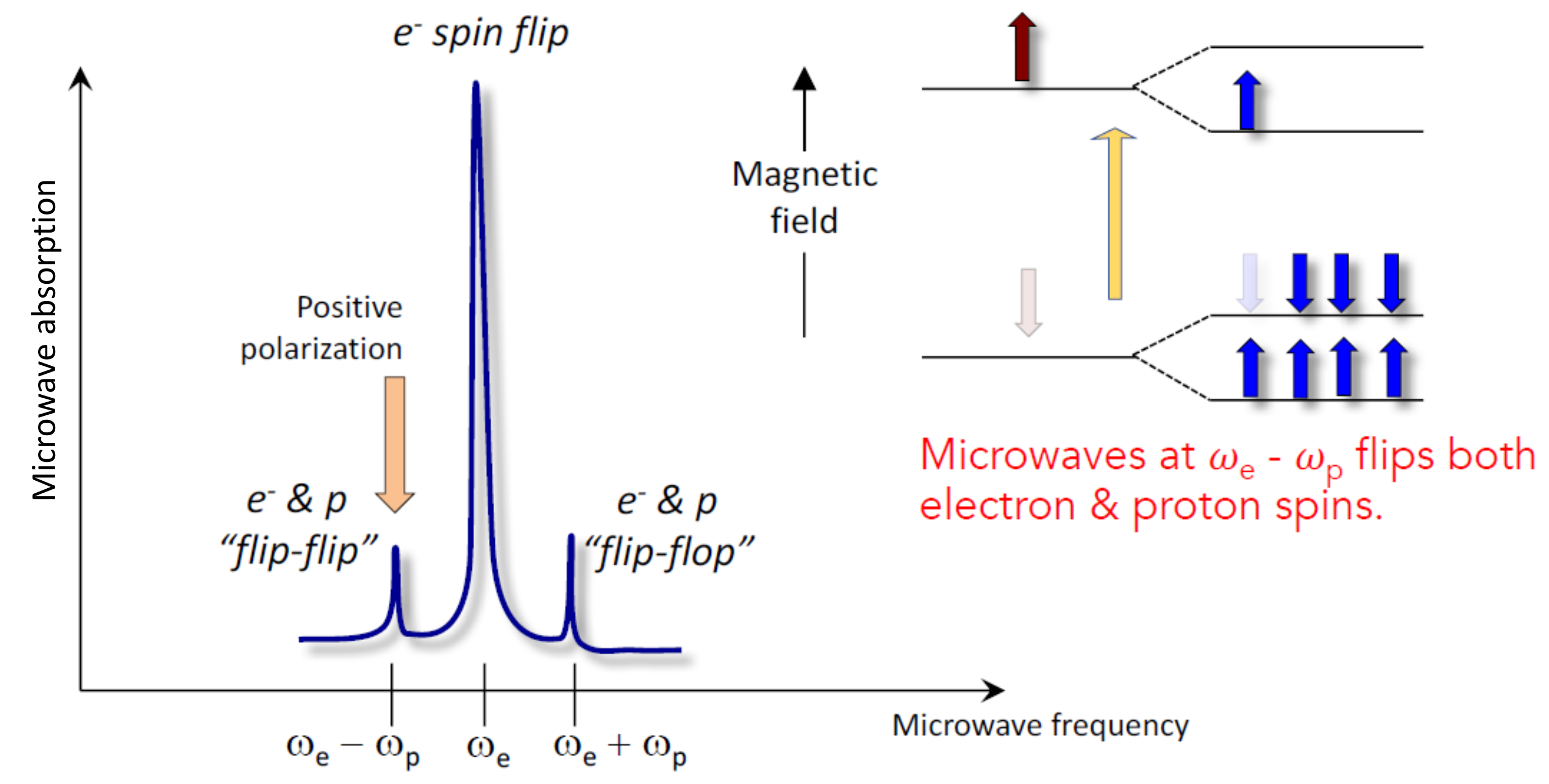

Courtesy of Chris Keith 


\section{Dynamic Nuclear Polarization}

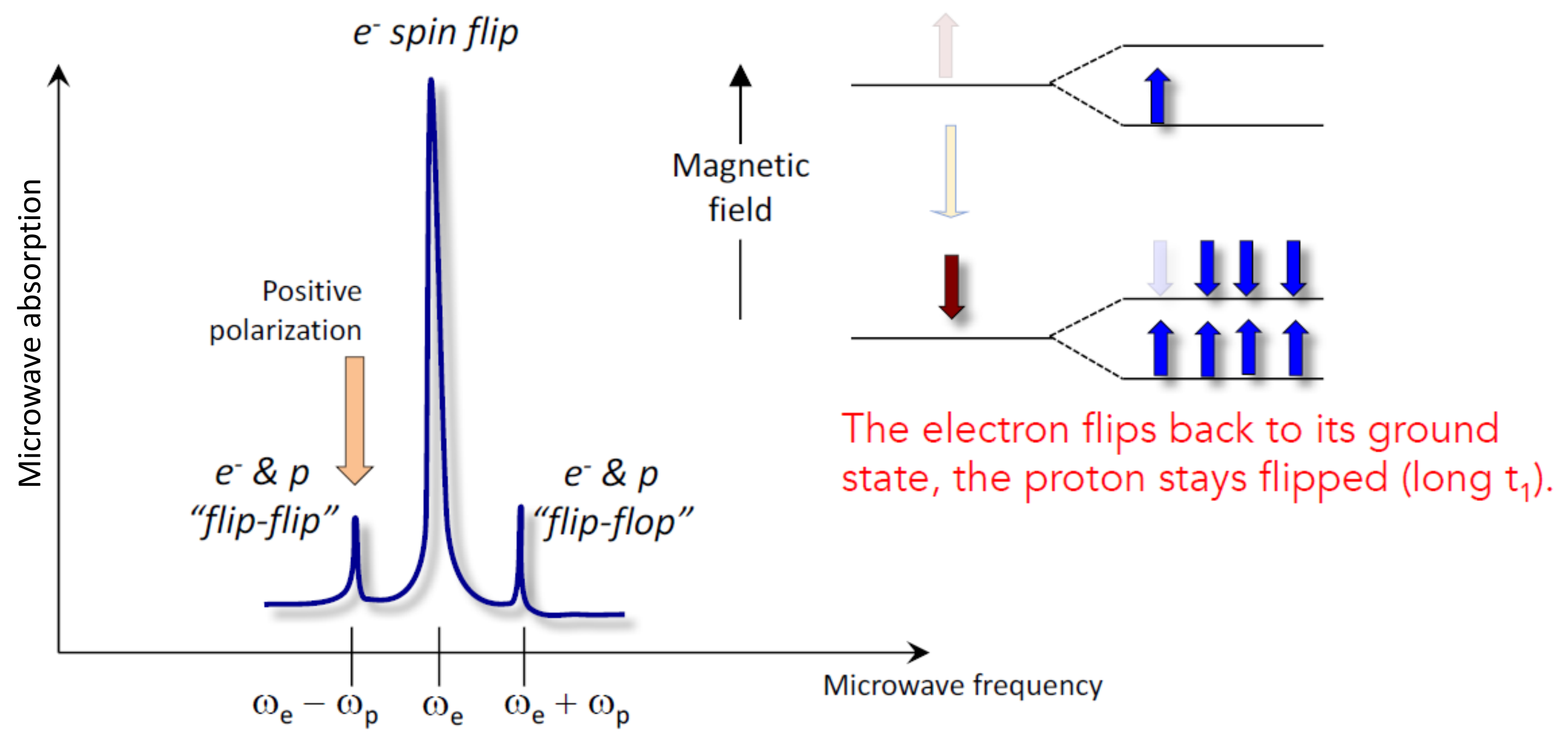




\section{Dynamic Nuclear Polarization}

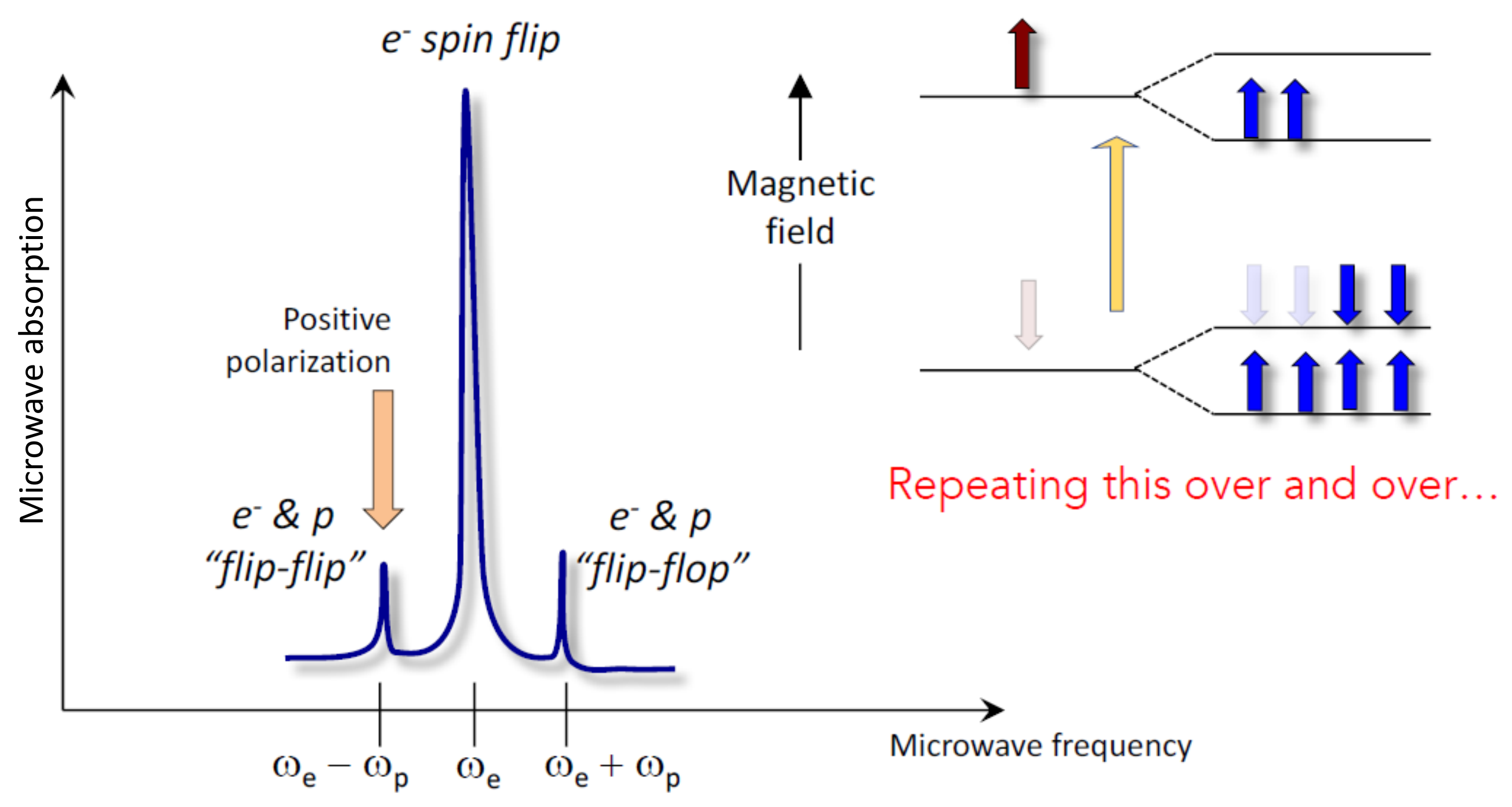

Courtesy of Chris Keith 


\section{Dynamic Nuclear Polarization}

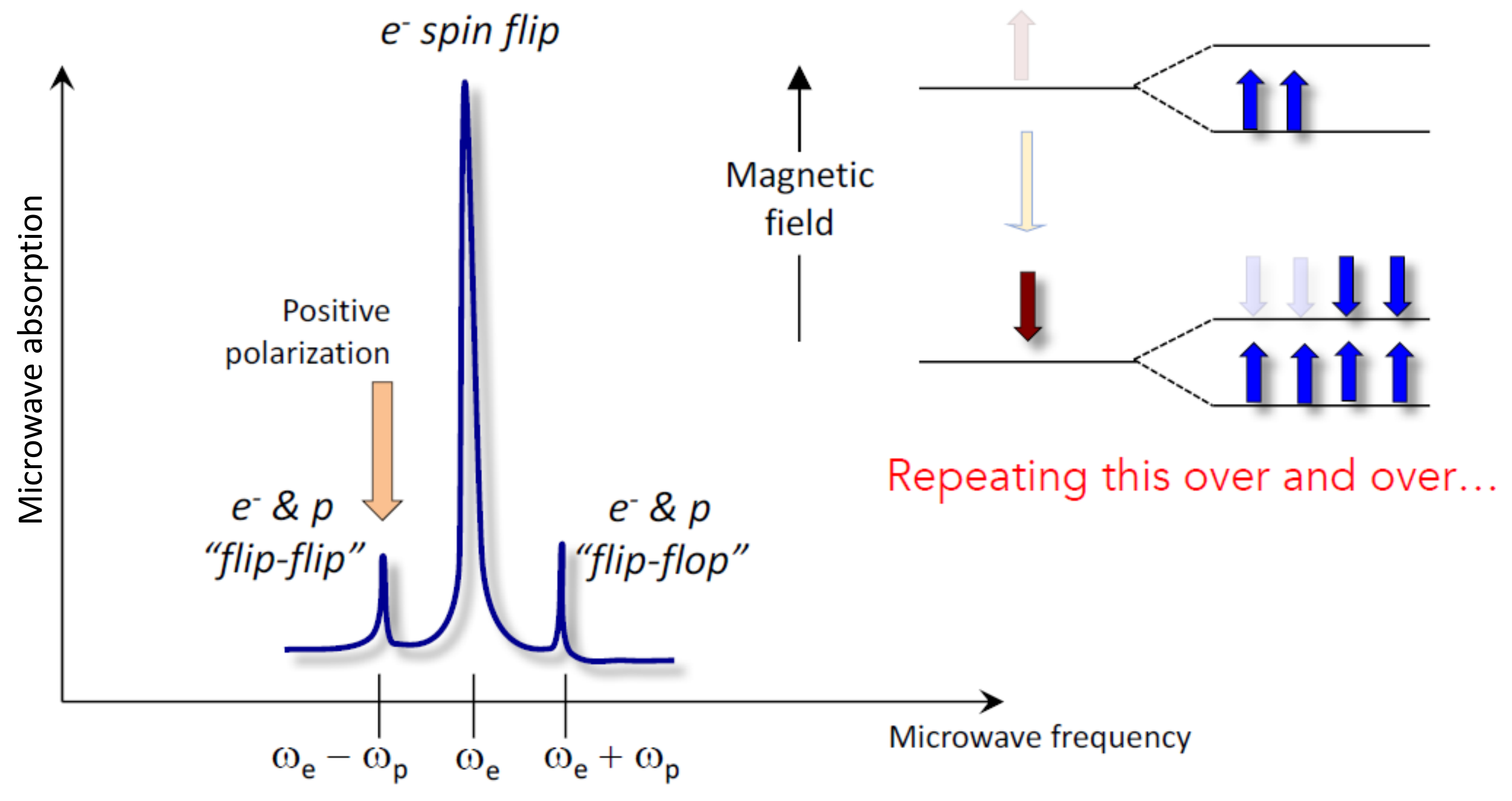

Courtesy of Chris Keith 


\section{Dynamic Nuclear Polarization}

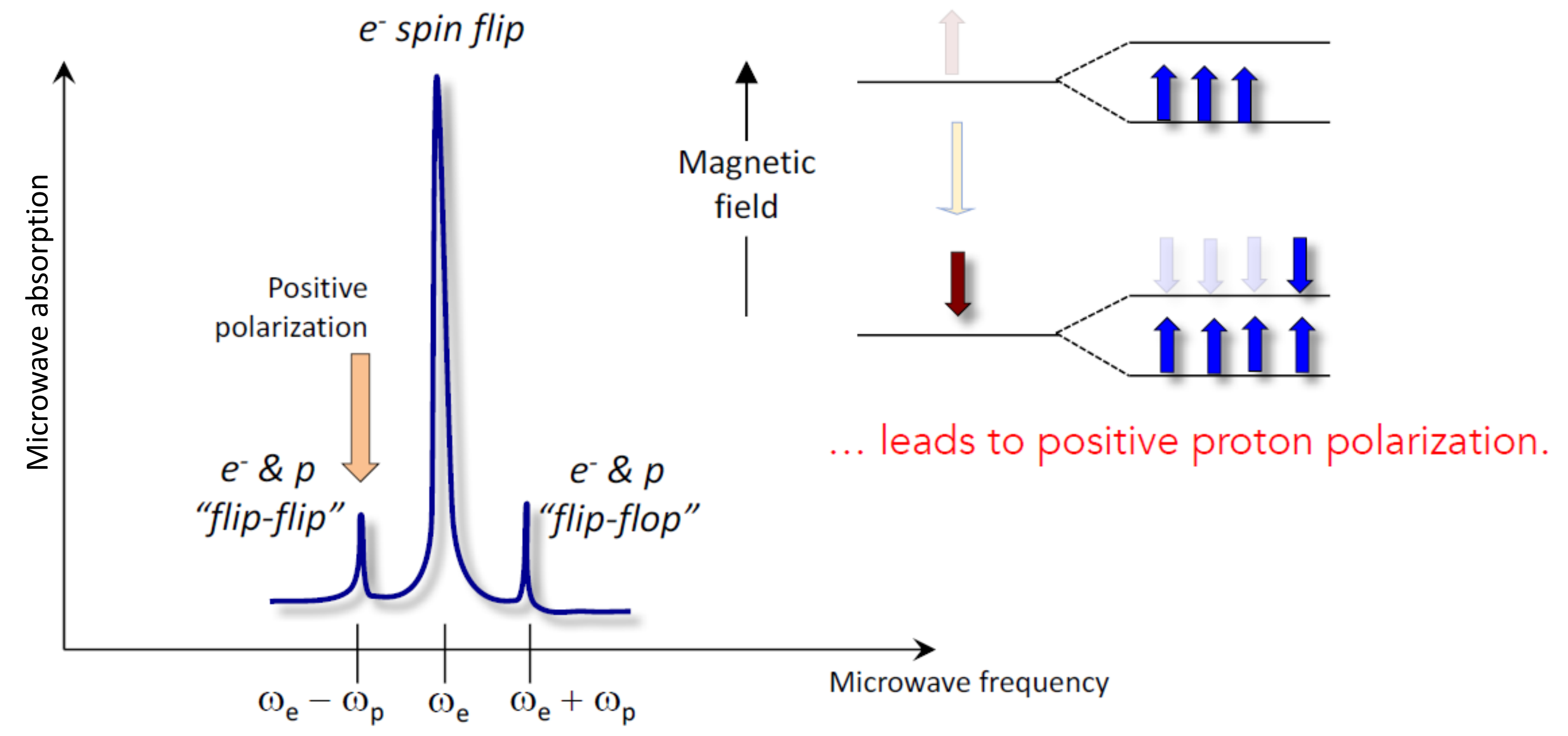

Courtesy of Chris Keith 


\section{What do we need to achieve significant proton polarization using the DNP Method?}

$\square$ Continuous microwaves generator

$\square$ Target material with a suitable number of unpaired electrons, resistance to radiation and reasonable dilution factor

$\square$ Superconducting magnet with homogenous fields in the target region

$\square$ Cryogenics system with high cooling power

$\square$ Reliable Nuclear-Magnetic Resonance (NMR) system for polarization measurement 


\section{Firsts for Polarized Taraets}

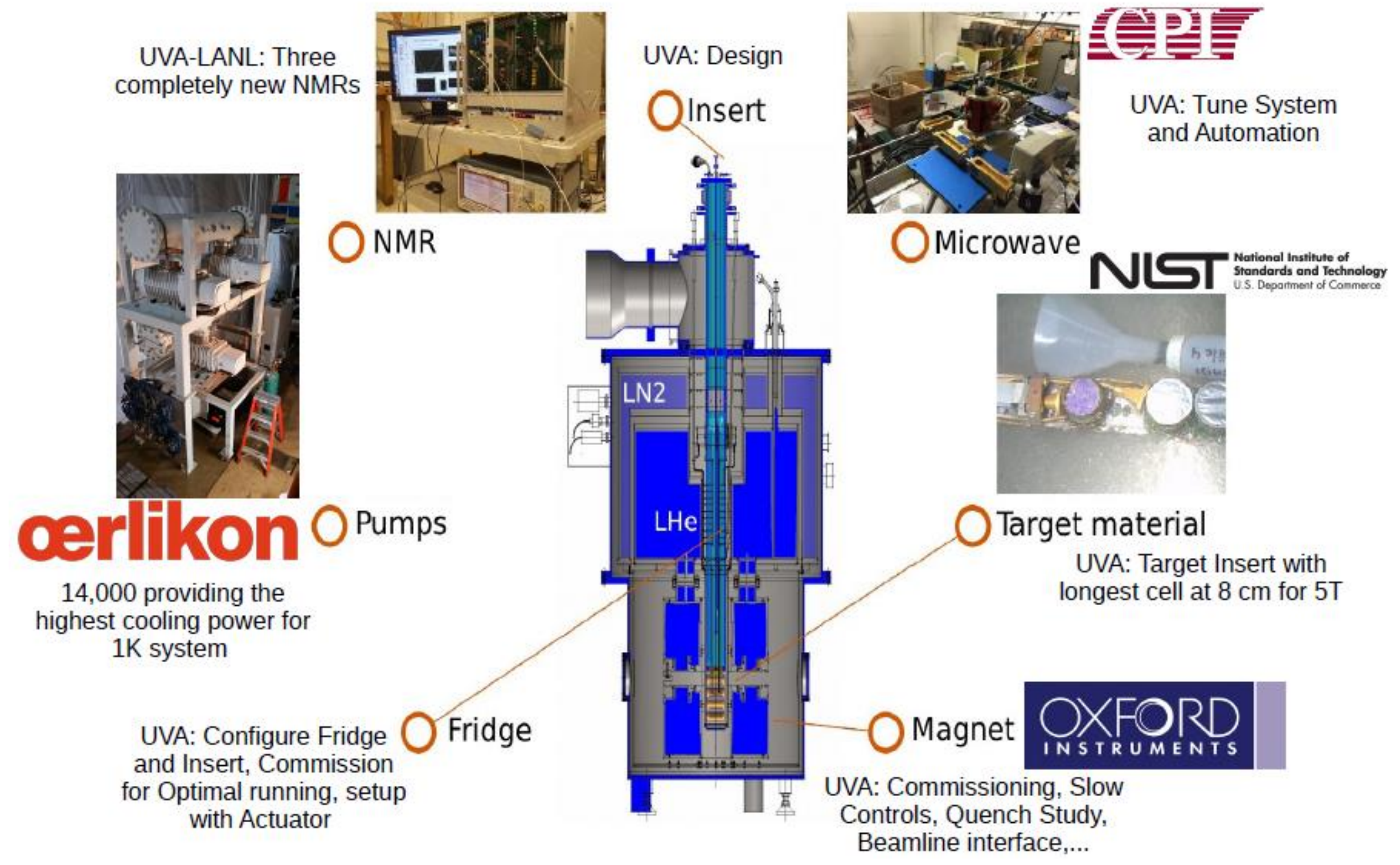




\section{$\square$ Microwave system}

$140 \mathrm{GHz}$ RF signal is generated by Extended-Interaction Oscillator (EIO) through interaction between electron beam (produced from $\sim \mathrm{kV}$ of cathode/anode) and resonant cavities
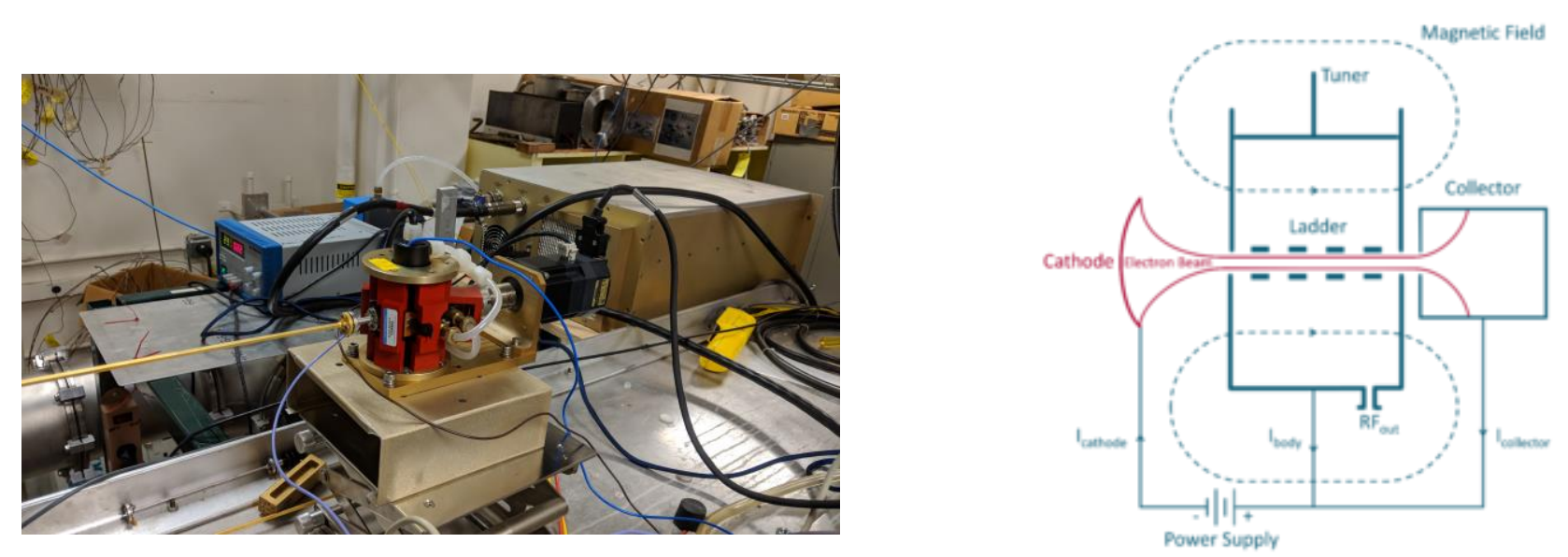

The optimal frequency changes as the target accumulate radiation damage from the beam. Therefore, the frequency is adjusted by adjusting the cavity size using a stepper motor ( $2 \%$ adjustment) 


\section{$\square$ Microwave system}

The EIO is coupled to the target cups via a wave-guide which send the microwave through the target stick terminating at a gold plate copper horn

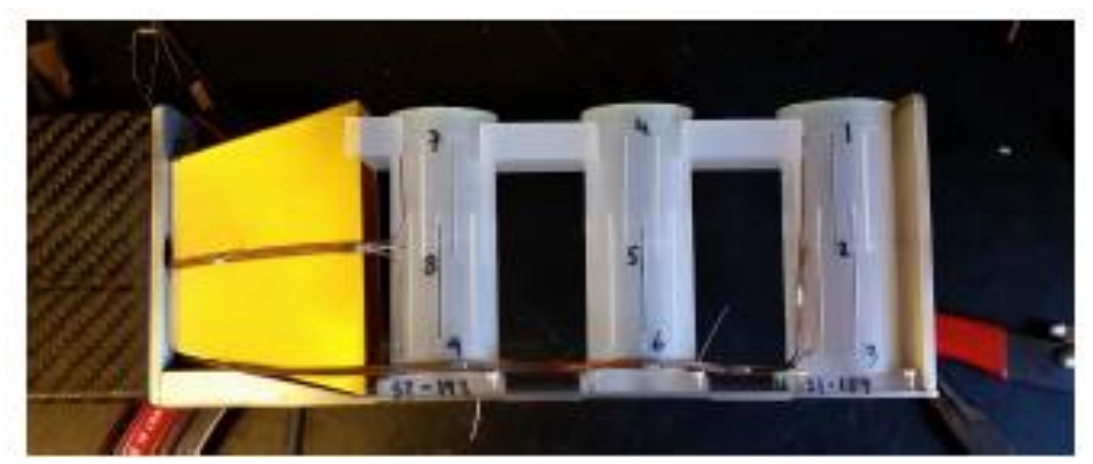

Other subsystems: Power supply, EIP frequency counter and microwave chiller. 


\section{$\square$ Target materials}

Target material for DNP characterized by

- Maximum achievable polarization

- Dilution factor

- Resistance to radiation damage

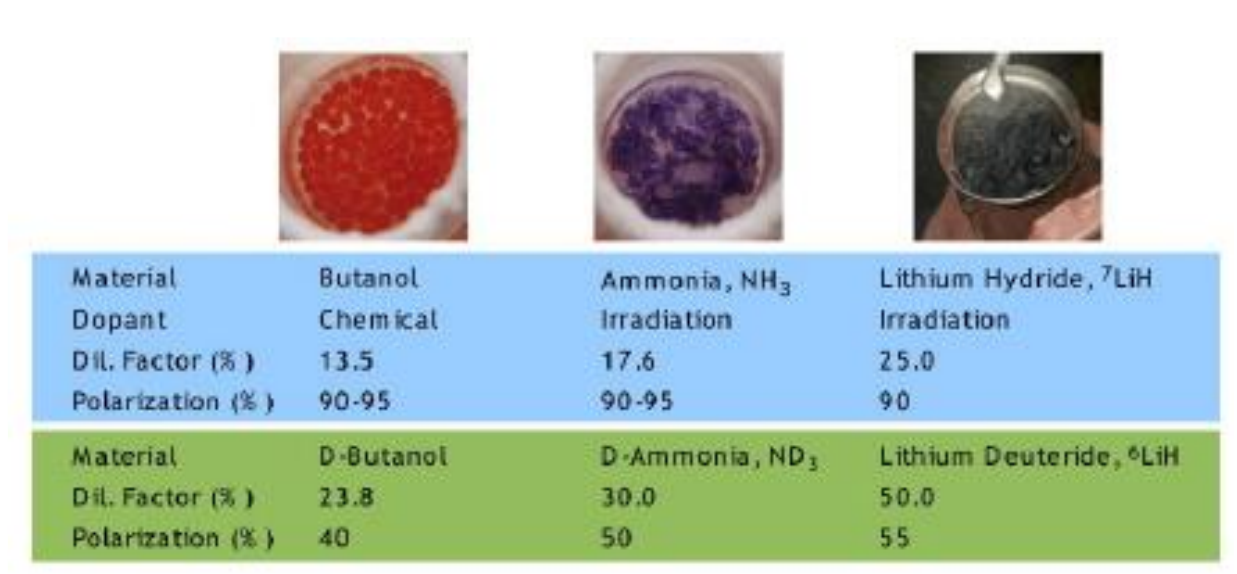

SpinQuest experiment will use $8 \mathrm{~cm}$ of solid $\mathrm{NH}_{3} / \mathrm{ND}_{3}$ as target materials which are doped with paramagnetic free-radical by being irradiated at NIST

The polarization decays over time due to the radiation damage and restored temporarily by annealing process (target is heated at $70-100 \mathrm{~K}$ ).

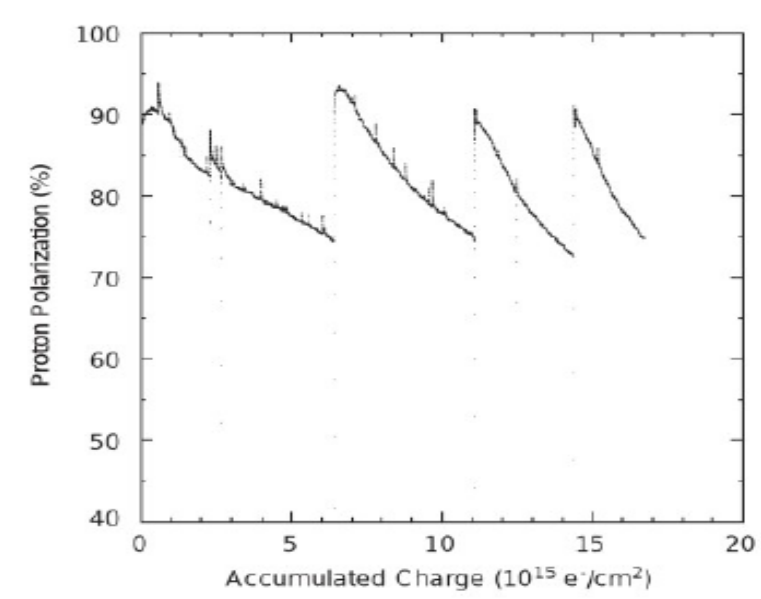




\section{$\square$ Superconducting-magnet system}

The superconducting magnet coils provide $5 \mathrm{~T}$ of transverse field in the target area with the homogeneity level of $10^{-4}$

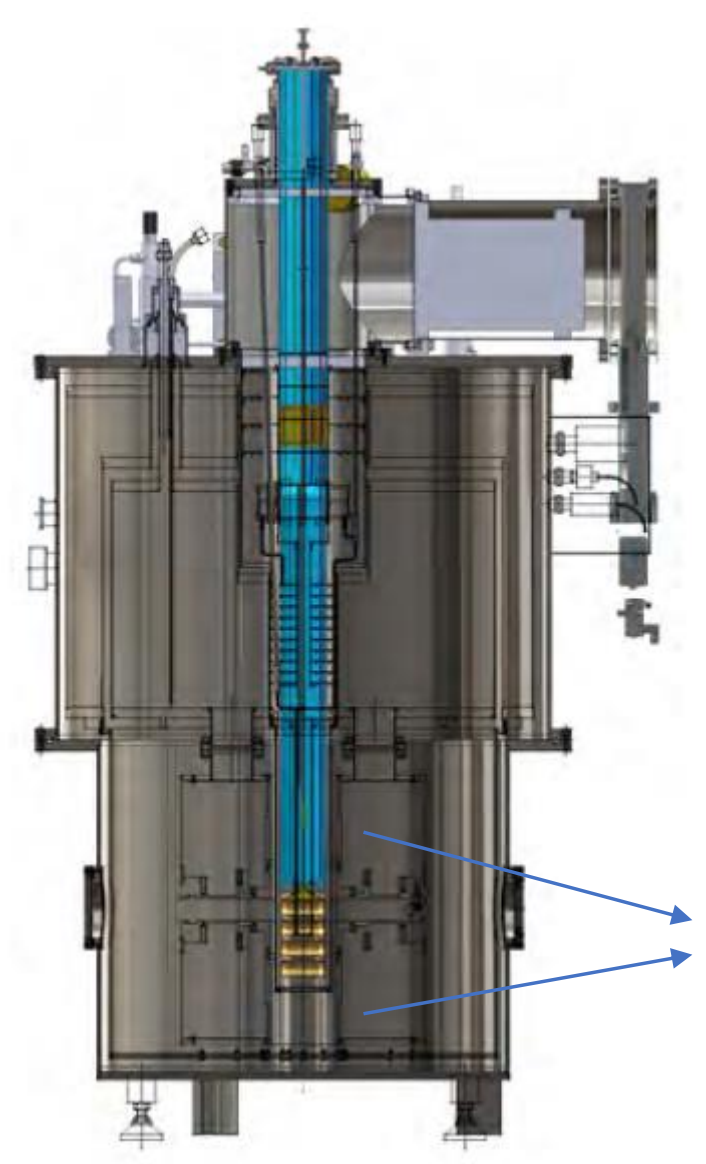

The NbTi coils are impregnated in epoxy to prevent them from moving during when the magnet is energized

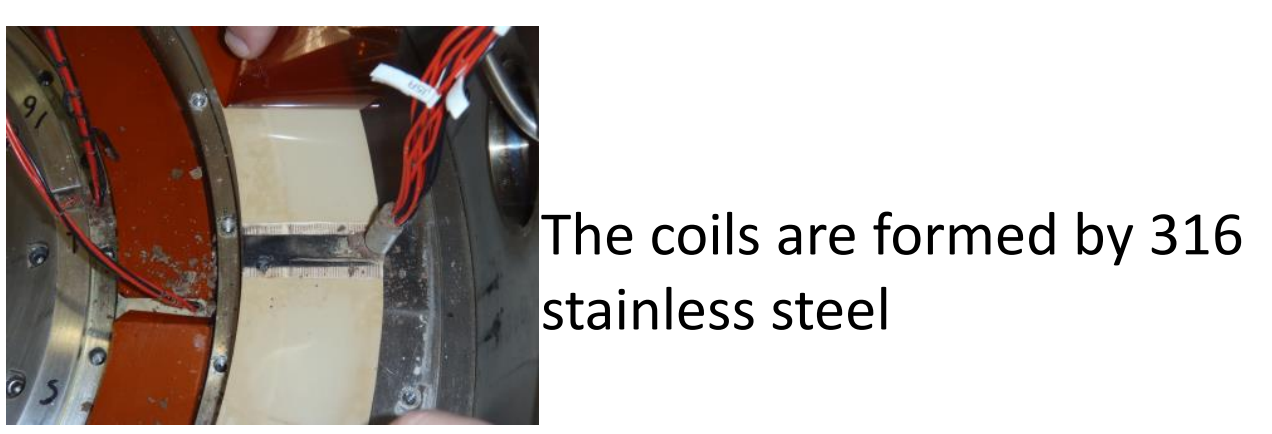




\section{$\square$ Superconducting-magnet system}

What is the maximum intensity of the proton beam before quenching the superconducting magnet?

The Thermal processes within the magnet is described by a general heat transfer equation:

$c \frac{\partial T}{\partial t}=\nabla(\kappa \nabla T)+P_{e x t}+P_{H e}$

$P_{\text {ext }}$ is the external-heat sources coming mainly from the beam-target

interactions

$P_{H e}$ is the heat transferred to the liquid Helium
The heat deposited to the magnet $\left(P_{\text {ext }}\right)$ is simulated using Geant:

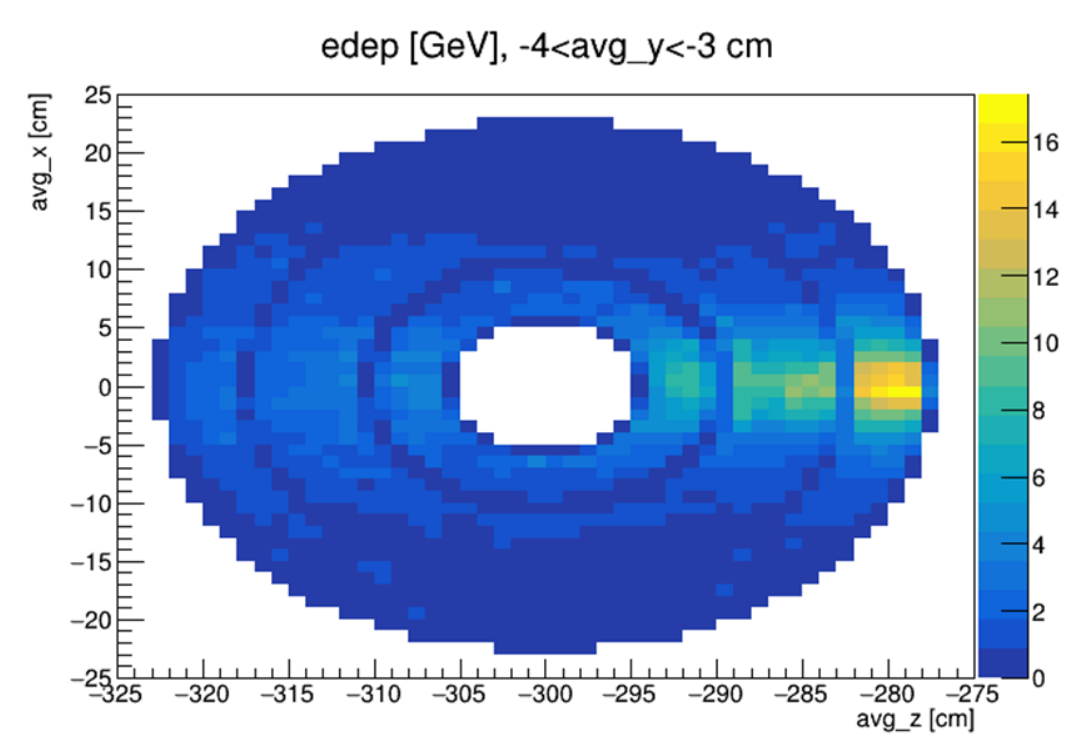

We will run at the highest instantaneous intensity for the polarized target with proton beam 


\section{$\square$ Superconducting-magnet system}

What is the maximum intensity of the proton beam before quenching the superconducting magnet?

The simulation was done using COMSOL

by applying Finite-Element Method

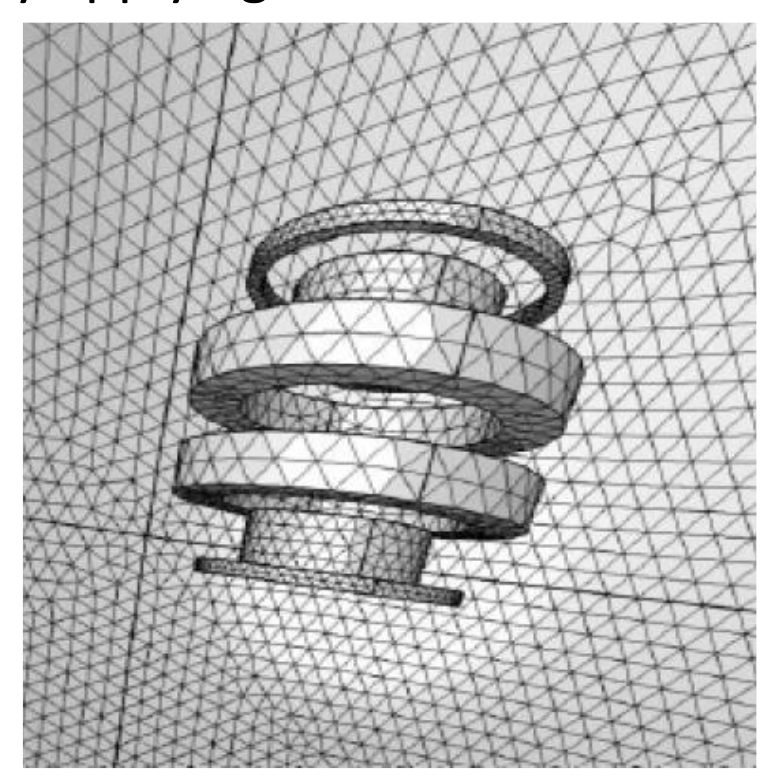

We obtained the spatial \& temporal profile of the temperature in the magnet

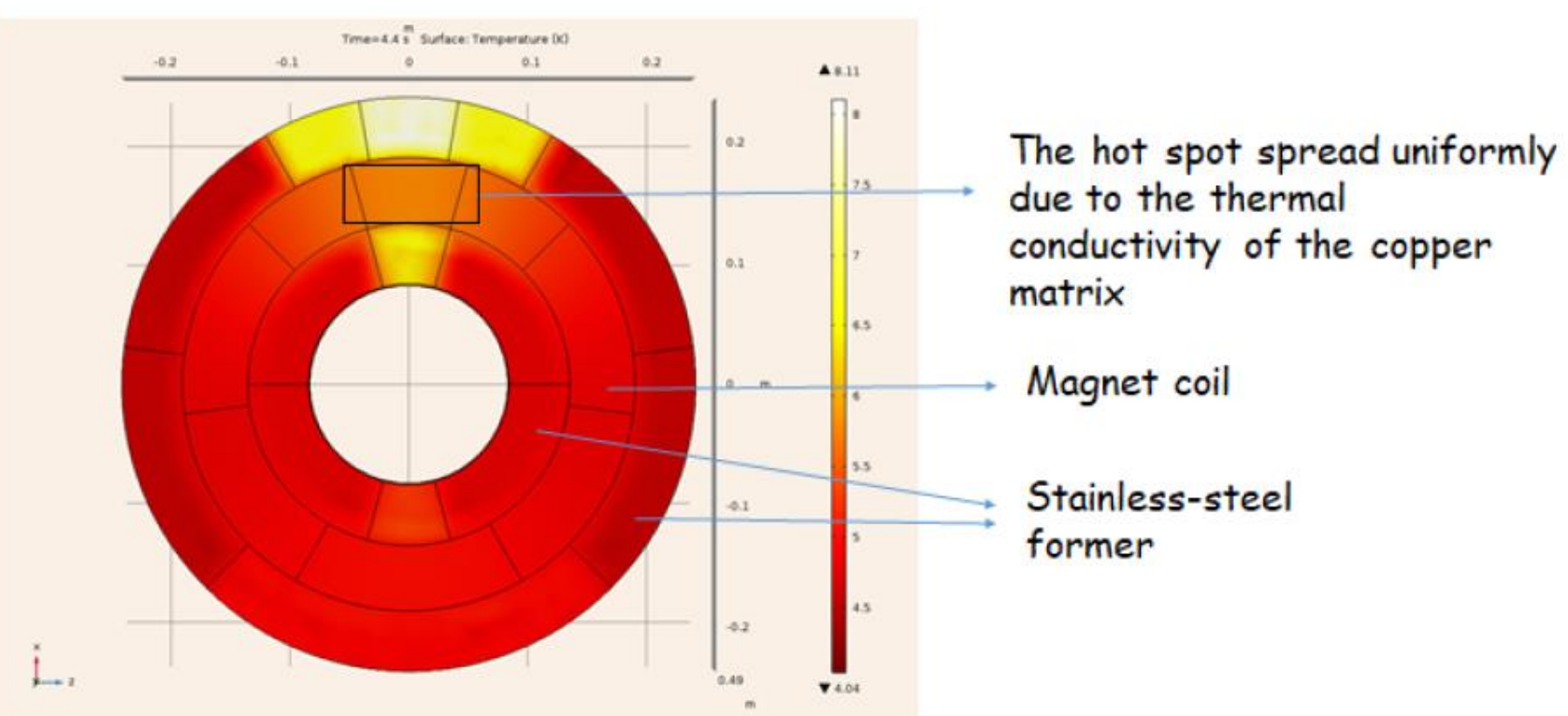

Based on this study the maximum intensity of the beam is $2.7 \times 10^{12}$ proton/sec (with pumping on the He reservoir) notes -> put error 


\section{$\square$ Cryogenics:}

\section{Evaporation Refrigerator with $3 \mathrm{~W}$ of Maximum Cooling Power}

Evaporated He from the target nose need to be pumped out by high powered pump to keep the temperature at $1 \mathrm{~K}$ at 0.12 Torr

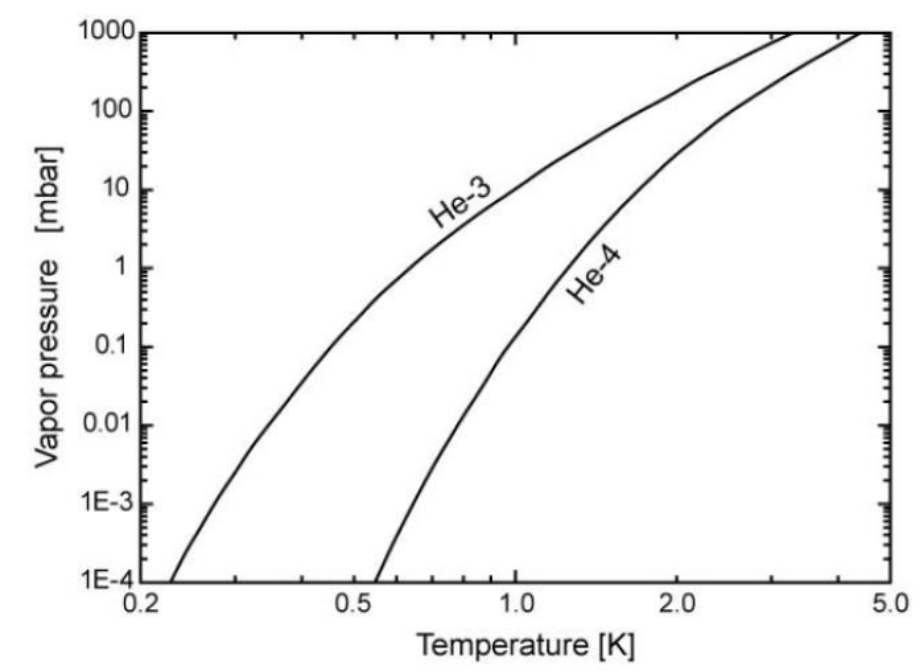

He Pressure-Temperature diagram
Critical components for high-cooling power refrigerator:

- High-power pump

- Sufficient supply of the liquid Helium

- Heat exchanger that bring the He temperature down from $4.2 \mathrm{~K}$ to $1 \mathrm{~K}$

- Thermal shielding 

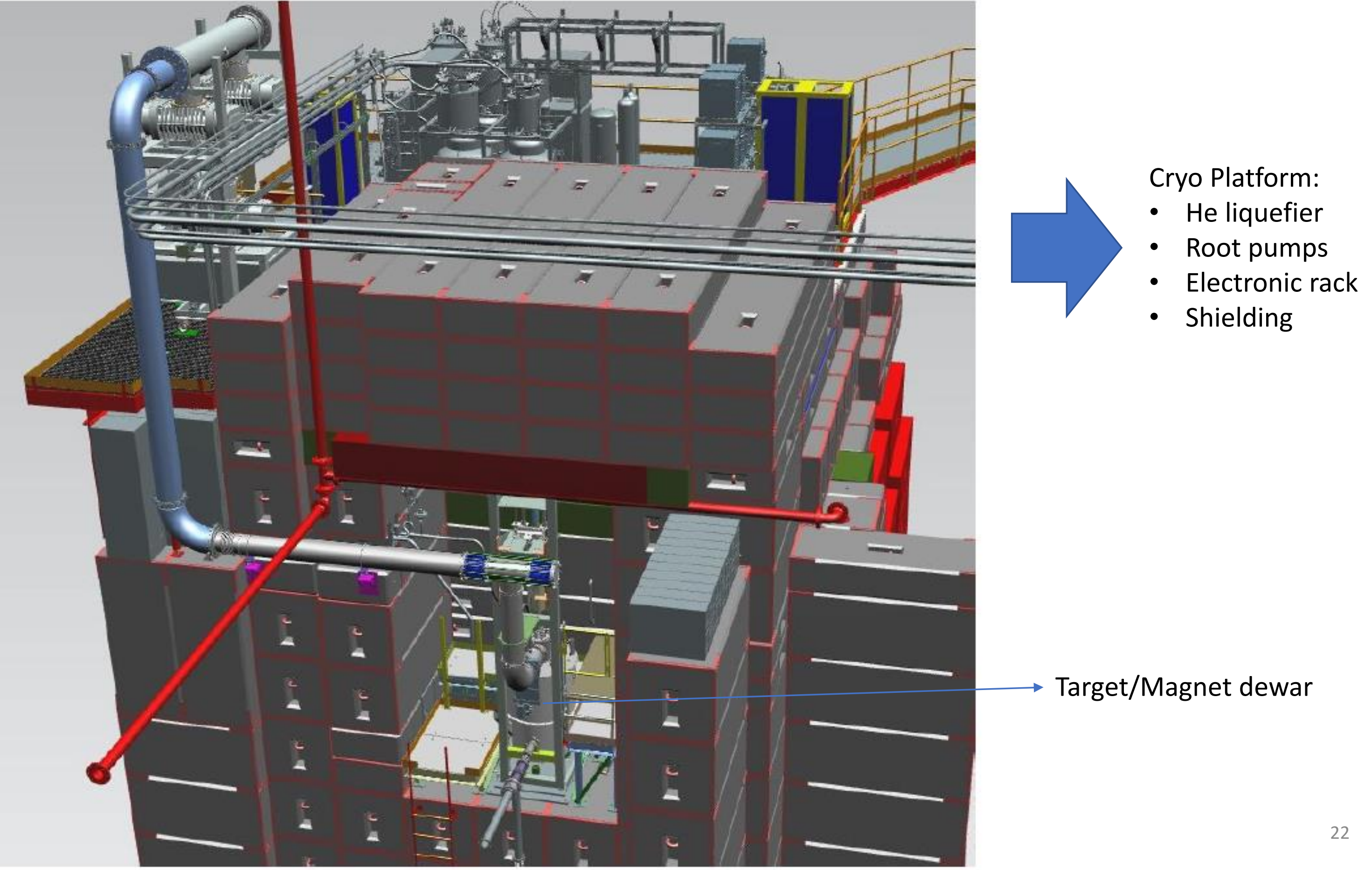

$\longrightarrow$ Target/Magnet dewar 


\section{$\square$ Cryogenics:}

Evaporation Refrigerator with 3 W of Maximum Cooling Power
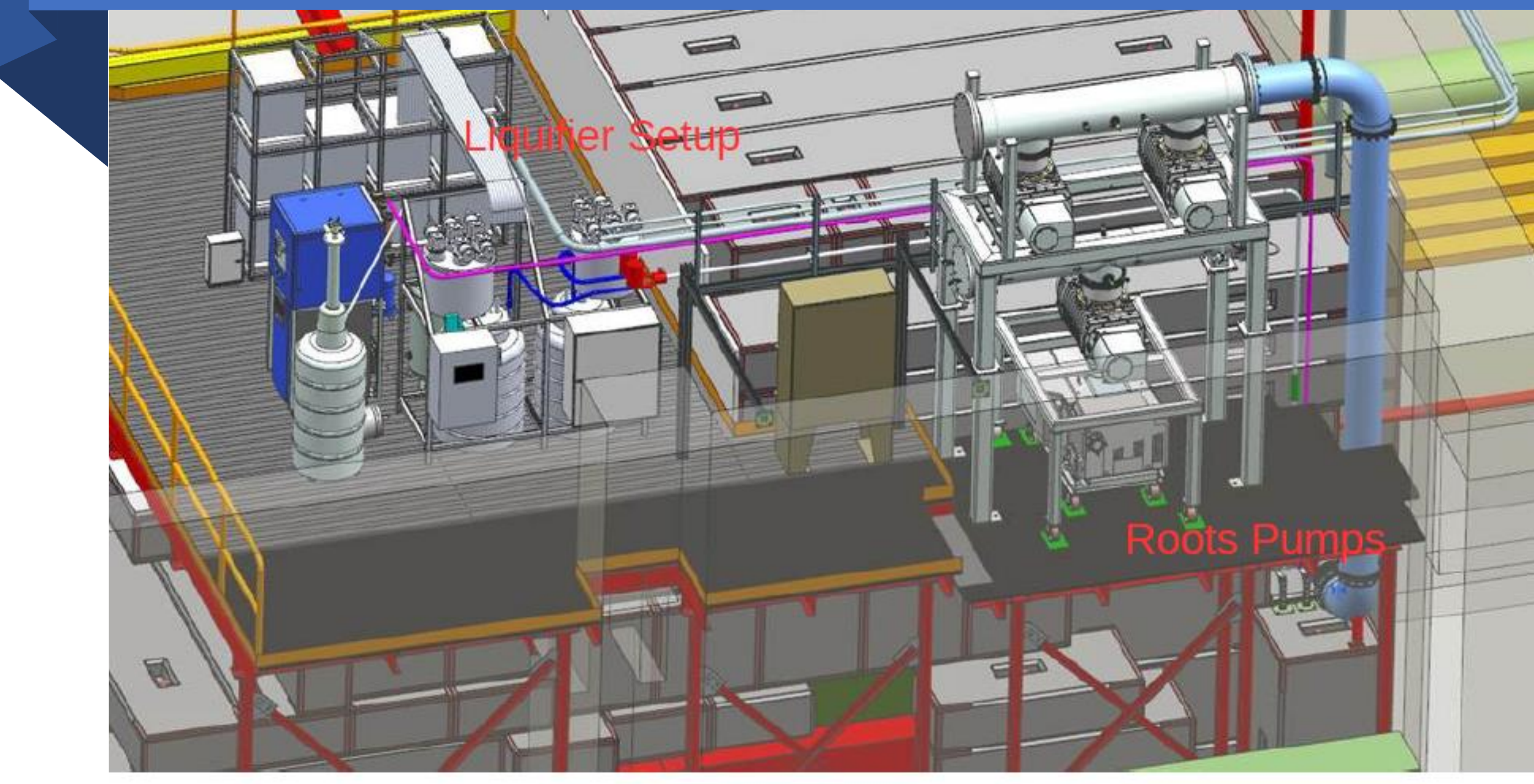

Cryo platform showing the He-liquefier and Roots pumps setup

The liquefier is capable to supply 200 liter per day of liquid He

Roots pump have the pumping capacity of $17 \mathrm{~K} \mathrm{~m}^{3}$ per hour 


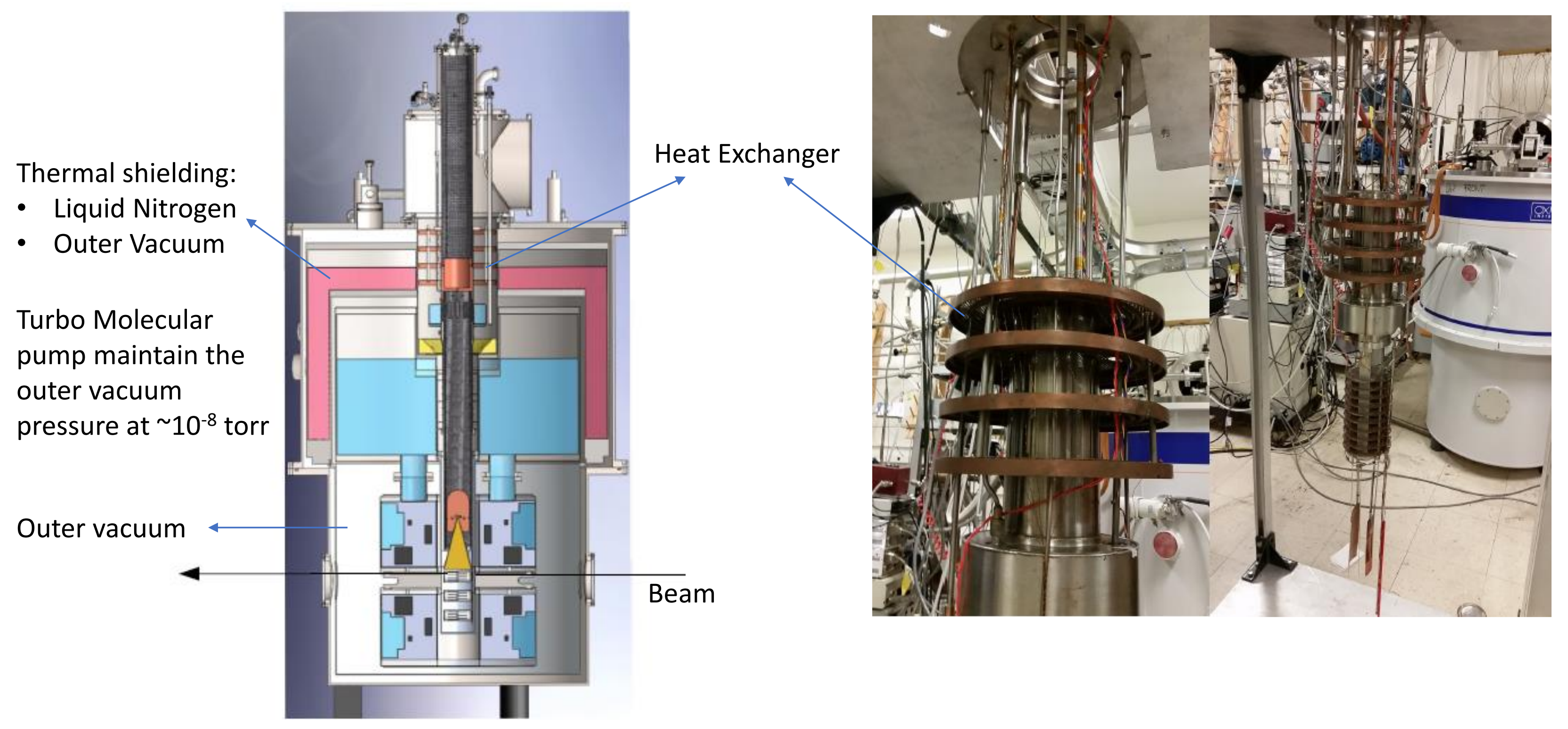




\section{$\square$ Nuclear Magnetic Resonances (NMR)}

Polarization of the proton is measured using NMR technique

An RF field at the Larmor frequency of the proton ( 213 $\mathrm{MHz}$ at $5 \mathrm{~T}$ ) can cause a flip of the spin

The RF field is produced by 3 NMR coils inside the target cup

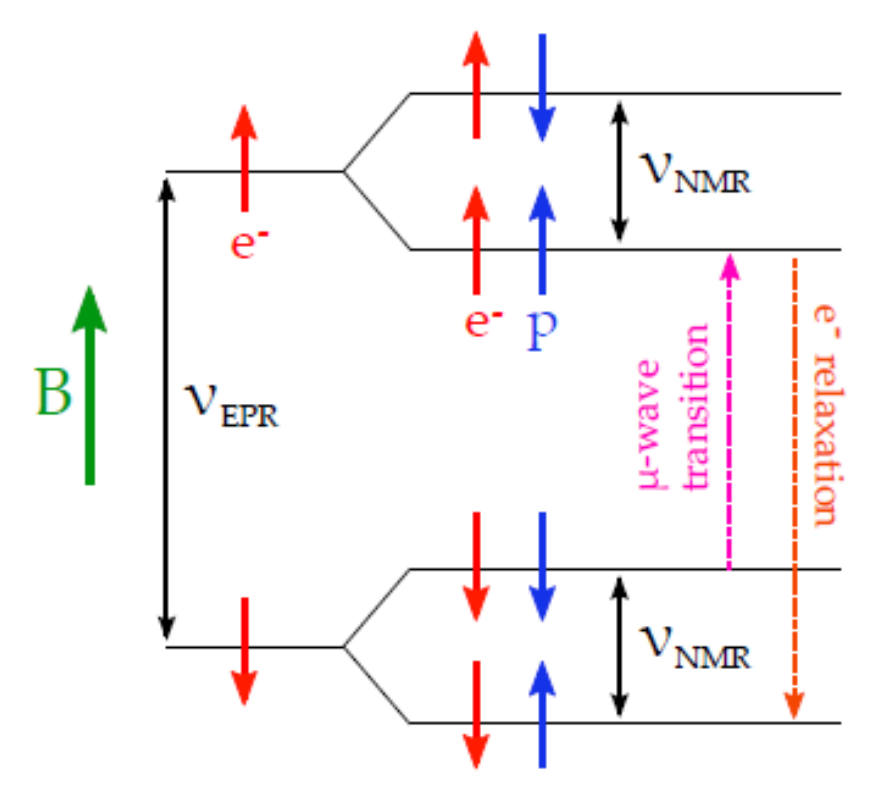

An RLC Circuit is tuned to the Larmor frequency of the target materials

The power generated or absorbed due to spin flip change the circuit impedance that can be observed

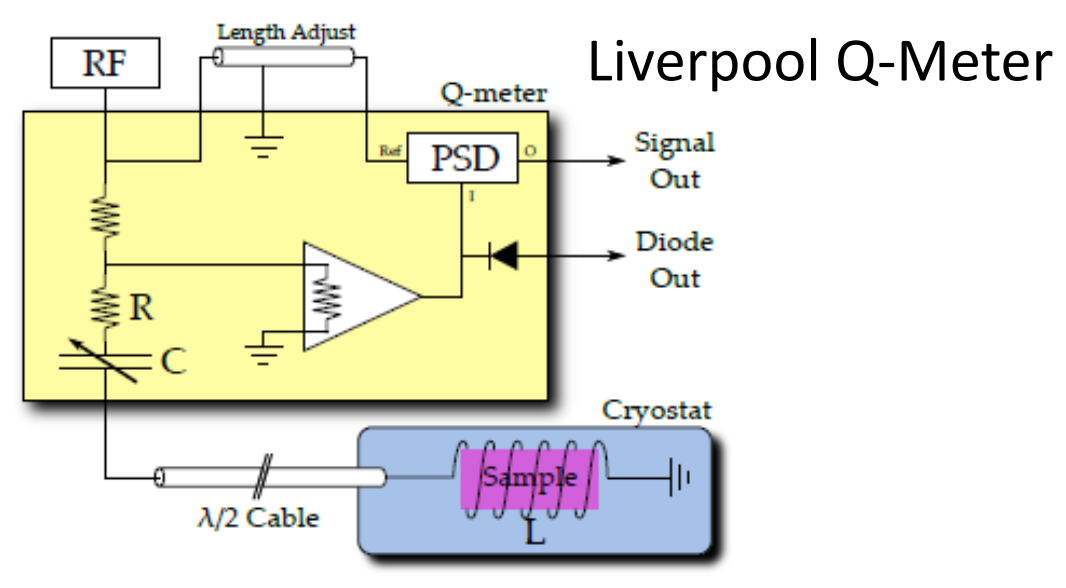

Courtesy of James Maxwell 


\section{$\square$ Nuclear Magnetic Resonances (NMR)}

Q-Curve is produced by sweeping the RF around the Larmor frequency

The signal area after background subtraction is proportional to the polarization

The proportional constant is obtained at ThermalEquilibrium measurement $P=\tanh \left(\frac{\mu B}{k T}\right)$
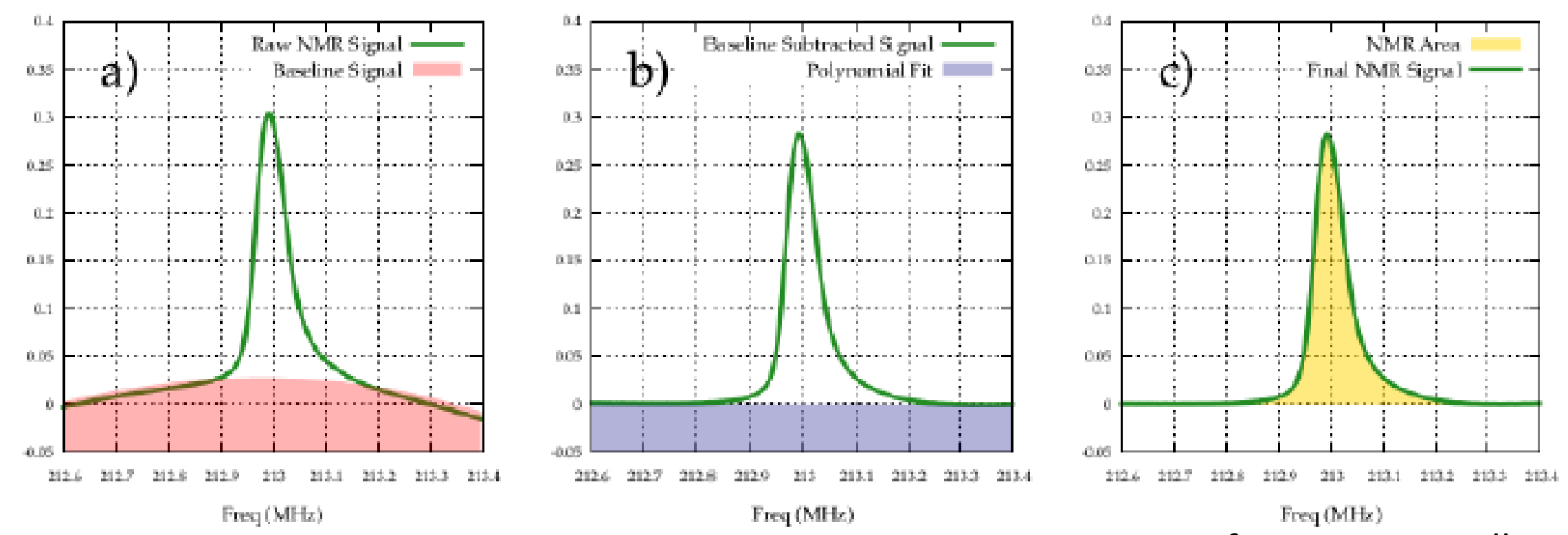

Courtesy of James Maxwell

Notes: SpinQuest experiment will use a new NMR system developed by LANL-UVA based on the original Liverpool Q-meter design 


\section{Summary}

The main polarized-target system for the SpinQuest experiment consist of a $5 \mathrm{~T}$ superconducting-split magnet, $140 \mathrm{GHz}$ RF generator, $8 \mathrm{~cm}$ of solid $\mathrm{NH}_{3} / \mathrm{ND}_{3}$ target, evaporation refrigerator and LANL/UVA-NMR system

During cooldowns at University of Virginia, The SpinQuest-polarized target achieved proton polarization of 95\% using Dynamic-Nuclear Polarization (DNP) technique 


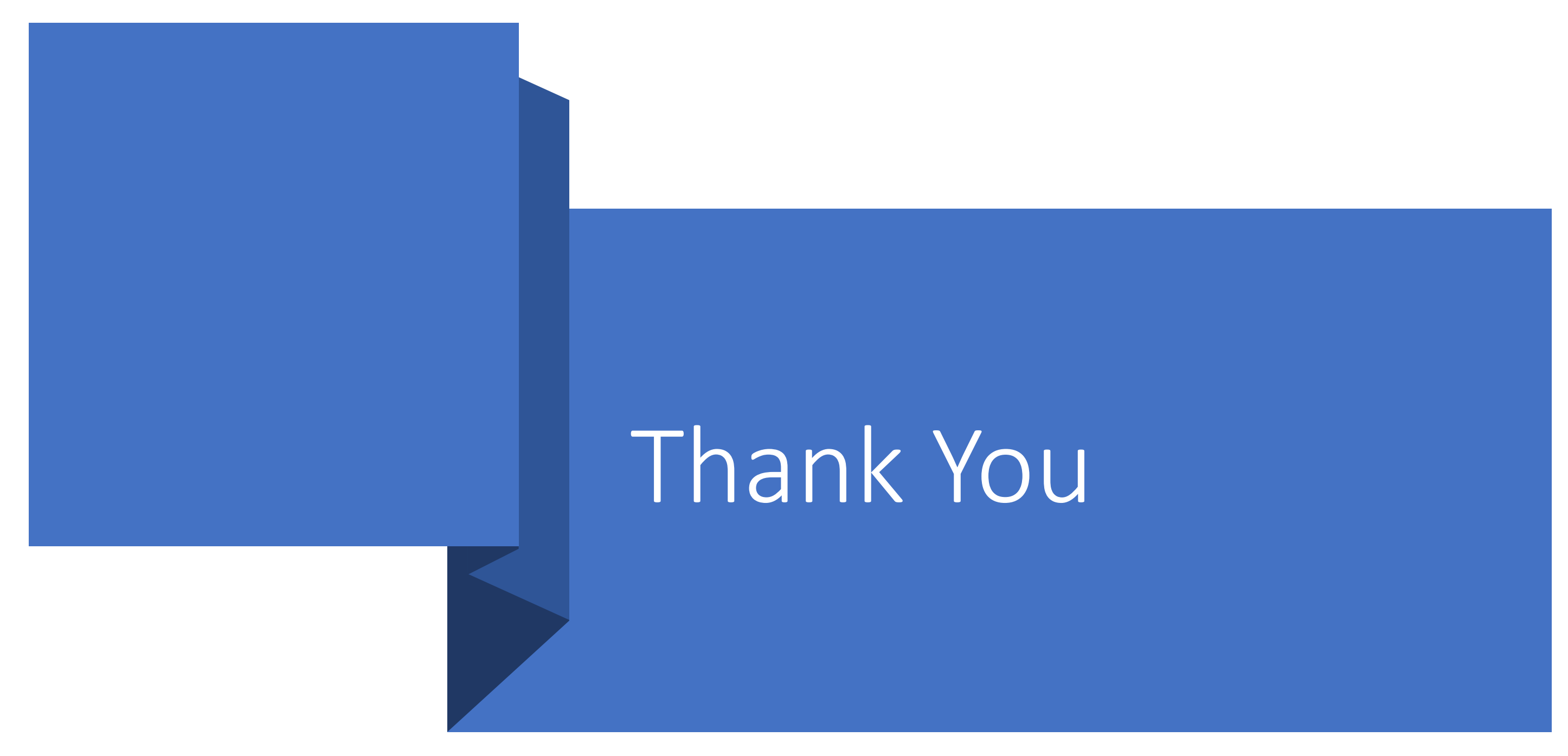

